\title{
THE ANTI- INFLAMMATORY EFFECTS OF SPINACIA OLERACEA LEAF EXTRACT ON CARRAGEENAN INDUCED INFLAMMATION IN RATS
}

\author{
YESMIN $\mathrm{S}^{1}$, RAHMAN KA ${ }^{2}$, AFROZ $\mathrm{R}^{3}$, PARVIN $Z^{4}$, LOTUS MA ${ }^{5}$, YEASMIN N ${ }^{6}$, MOON KJ $^{7}$, \\ AHMED MAU ${ }^{8}$
}

\begin{abstract}
Context: Use of herbal medicine throughout the world is increasing. Plants still remaining the primary source of supply of many important drugs used in modem medicine. Spinacia Oleracea i,e spinach leaves contain more number of anti-inflammatory phytochemcicals such as carbohydratie, tanins and phenolic compounds, saponins, flavinoids and steroid. Better collagenation seen under the influence of the flavinoids, phenolic compound and saponins which is responsible for the free radical scavenging activity and are believed to be some of the most important component for wound healing and antioxident activity. Spinacia Oleracea scavenges free oxygen redicals and increases the catalase level in granulation tissue. Plants still remaining the primary source of supply of many important drugs used in modem medicine. Considering its medicinal value and availability in our country this study was undertaken to evaluate the anti-inflammatory effect of the Spinacia Oleracea leaf extract in rat models.

Material and Methods: The experiments were carried out on 30 (thirty) Swiss male albino rats. They were collected from the ICDDRB, Dhaka. The rats were of male weighing between 150$200 \mathrm{gm}$ which were divided randomly into 5 groups each having 6 rats. Groups were labeled as group-I, group-II, group-III, group-IV and group-V. The anti inflammatory effect of Spinacia Oleracea leaf extract in experiment rat were evaluated and compared with the anti inflammatory effects of aspirin and hydrocortisone. The study was prospective experimental type and was conducted in the department of Pharmacology, Dhaka Medical College, Dhaka, from July 2011 to June 2012.

Result : Administration of mathanolic extract and water extract of Spinacia Oleracea leaf at a dose of $200 \mathrm{mg} / \mathrm{kg}$ body weight orally produced a significant $(P<0.05)$ anti-inflammatory effect, and the percentage of inhibition of oedema formation was $28.75 \%$ and $40.79 \%$ respectively. There were highly significant $(P<0.05)$ percentage of inhibition of oedema formation was observed in aspirin (40.52\%) and in hydrocortisone (47.71\%).
\end{abstract}

Conclusion: Spinacia Oleracea leaf extract, possess significant anti-inflammatory activity in rats.

Key words : Anti-inflammatory effect, Spinacia Oleracea leaf extract, Carrageenam.

J Dhaka Med Coll. 2018; 27(1) : 88-93

\section{Introduction:}

Inflammation is defined as the local response of living mammalian vascularized connective tissue to the injury caused by various exogenous and endogenous stimuli. It is a body defense reaction in order to eliminate or limit the spread of injurious agent as well as to remove the consequent necrosed cells and tissue ${ }^{1}$. Inflammation helps to clear the infections and along with the repair, it makes wound healing possible, both have considerable potential to cause harm. For example, inflammatory reactions underlie life threating anaphylactic responses to insect bites or drugs as well as, chronic licenses such as rheumatoid arthritis and atherosclerosis. An initial inflammatory stimulus triggers the release of chemical mediators from plasma or cells which then

1. Dr. Sultana yesmin, Assistant Professor, Department of Pharmacology. Dhaka Medical College, Dhaka

2. Dr. Kazi Afzalur Rahman, Associate Professor, Department of Pharmacology. Dhaka Medical College, Dhaka.

3. Dr. Rumana Afroz, Assistant Professor, Department of Pharmacology. Dhaka Medical College, Dhaka.

4. Dr. Zohora Parvin, Lecturer, Department of Pharmacology. Sher-E-Bangla Medical College, Barisal.

5. Dr. Mahbuba Jahan Lotus, Lecturer, Department of Pharmacology. Dhaka Medical College, Dhaka.

6. Dr. Nahid Yeasmin, Assistant Professor, Department of Physiology, Dhaka Medical College, Dhaka

7. Dr. Kawsar Jahan Moon, Lecturer, Department of Pharmacology. Dhaka Medical College, Dhaka.

8. Dr. Md. Ashraf Uddin Ahmed, Resident Physician, BIRDEM General Hospital, Dhaka

Correspondence : Dr. Sultana Yesmin, Assistant Professor (CC), Department of Pharmacology, Dhaka Medical College, Dhaka, Cell Phone:+8801715267403, E-mail: sultanavesminkhan@vahoo.com

Received: 10 May 2017

Revision: 26 August 2017

Accepted: 15 September 2017 
regulate the subsequent vascular and cellular rasponses ${ }^{2}$.

Use of herbal medicine throughout the world is increasing. Plants still remaining the primary source of supply of many important drugs used in modem medicine. Plants had been used for medicinal purposes long before recorded history. Ancient Chinese and Eqyption papyrus writing describe medicinal use of plants. African and Native American indigenous cultures used herbs as healing rituals, while others developed traditional medical systems (Ayurveda and Traditional Chinese medicine). Researcher found that people in different part of the world have a tendency to use same or similar plant for the same purpose. There are also many plants which posses reported anti-inflammatory activity like Curcuma longa, Cyperus rotundus, Glycyrrhiza glabra, Tylophora irtdica, Berbesis aristata, Mesua ferral and Psoralea corylifolia. For this reason the present study was selected to evaluate the antiinflammatory activity of Spinacia Oleracea in experimentally induced inflammation in rats.

Spinacia Oleracea i,e spinach leaves contain more number of anti-inflammatory phytochemcicals such as carbohydrate, tanins and phenolic compounds, saponins, flavinoids and steroid. Better collagenation seen under the influence of the flavinoids, phenolic compound and saponins which is responsible for the free radical scavenging activity and are believed to be some of the most important component for wound healing and antioxident activity. Spinacia Oleracea scavenges free oxygen redicals and increases the catalase level in granulation tissue. Plants still remaining the primary source of supply of many important drugs used in modern medicine.

The anti-inflammatory drugs that are now available including non-steroidal antiinflammatory drugs, corticosteroids, diseases modifying anti-rheumatic drugs such as methotrexate, cyclosporine etc. None of these drugs have been found safe, all are known to produce mild to serious side effect. Corticosteroids initially a drug of great promise can produce devastating side effect which include aseptic bone necrosis, thin skin and raised blood pressure. In the light of these facts, it can be stated that modem drugs have many adverse effects and moreover they can only control the disease, but cannot cure the same.
To avoid such problems and sufferings, people are becoming gradually interested to herbal medicine throughout the world because of their easy available and cost effectiveness as well as lack of any significant side effects. As our knowledge goes no other work has been done on the both acute and chronic antiinflammatory effect of Spinacia Oleracea or spinach in our country. Considering its medicinal value and availability in our country this study was undertaken to evaluate the antiinflammatory effect of the Spinacia Oleracea leaf extract in rat models. In this study acute inflammation were tested and antiinflammatory effects of Spinacia Oleracea leaf were compared with both steroidal and non steroidal anti-inflammatory agents.

\section{Materials and Methods:}

The present study was performed on 30 (thirty) adult male albino rats weighing $200 \pm 10 \mathrm{gm}$. The animals were given standard rat feed and water ad libitum. They were kept in the laboratory environment for seven days and fasted overnight and weighed before the experiment. The animals were randomly divided into 5 groups consisted of 6 rats in each group. Groups were labeled as group-I, group-II, group-III, group-IV and groupV. The leaf extract both methanolic aqueous fraction and water extract of Spiracia Oleracea were given orally by nasogastric tube at doses of $200 \mathrm{mg} / \mathrm{kg}$ body weight. Aspirin were given orally by nasogastric tube at a dose of $100 \mathrm{mg} / \mathrm{kg}$ body weight and Hydrocortisone were given subcutaneously at a dose of $2 \mathrm{mg} / \mathrm{kg}$ body weight as standard anti-inflammatory drugs. One group of rats given only saline solution were served as control. After one hour of drug administration, $0.1 \mathrm{ml}$ of $1 \%$ Carrageenan in sterile saline solution were injected into the sub-plantar surface of the right hind paw for the production of acute inflammation. Anteroposterior diameters of paw were measured by slide calipers after 1, 2, 3 hours of Carrageenan injection. Progress of the local inflammatory exudative lesion was assessed by measuring the maximum linear cross-section of the joint ${ }^{3} 1$ hour before and 3 hours after Carrageenan injection into the rats paw in the control, extract and drugs treated grups.

The percent inhibition of oedema formation was calculated by using the formula :

Percent inhibition $=\frac{\mathrm{vc}^{-}}{\mathrm{vtvc}}$ 
Where 'c' represents anteroposterior diameter of the paw of the control group and ' $t$ ' represents the average anteroposterior diameter of paw of the test group respectively ${ }^{4}$.

\section{Materials:}

Mathanolic extract of Spiracia Oleracea Water extract of Spiracia Oleracea Acetyl salicylic acid (aspirin)

Hydrocortisone

$1 \%$ Carrageenan solution/ suspension in normal saline. Normal rat food

\section{Grouping of the animals :}

The animals were divided into five groups. Inflammation was produced by injecting $0.1 \mathrm{ml}$ of a $1 \%$ Carrageenan suspension in normal saline in all experimental animals and treated as follows

Group I : Consist of 6 rats and served as control that received normal saline in a volume of $0.6 \mathrm{ml}$ one hour before Carrageenan injection.
Group II : Consist of 6 rats and recieved methanolic aqueous fraction of Spinacia Oleracea leaf extract at a dose of $200 \mathrm{mg} / \mathrm{kg}$ body weight orally one hour before the Carrageenan injection.

Group III : Consist of 6 rats. The animals received aqueous extract of Spinacia Oleracea leaf at a dose of $200 \mathrm{mg} / \mathrm{kg}$ body weight orally one hour before the Carrageenan injection.

Group IV : Consist of 6 rats. The animals received aspirin at a dose of $100 \mathrm{mg} / \mathrm{kg}$ body weight orally one hour before the Carrageenan injection.

Group V : Consist of 6 rats. The animals recieved hydrocortisone at a dose of $2 \mathrm{mg} / \mathrm{kg}$ body weight one hour before the Carrageenan injection.

The study was prospective experimental type and was conducted in the Dept. of Pharmacology, Dhaka Medical College, Dhaka, from July 2011 to June 2012. All the data were analyzed by SPSS version 16.1. Significance of difference between groups were assesed by using ANOVA test.

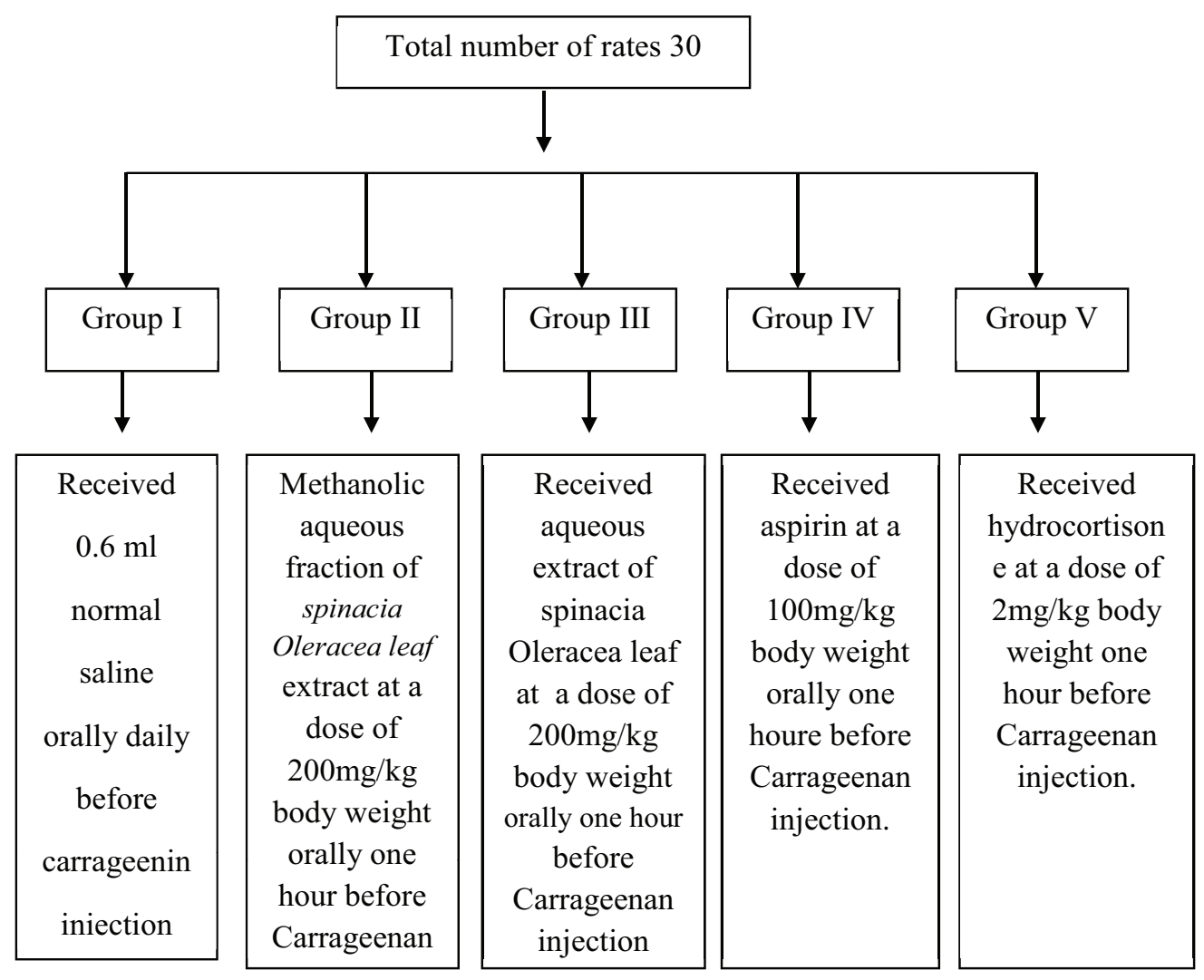

After 3 hours of Carrageenan injection mean increase in the anteroposterior diameter of rats paw oedema were measured

Fig.-1 : Outline of the study 
The Anti- Inflammatory Effects of Spinacia Oleracea Leaf Extract on Carrageenan Induced Inflammation Yesmin S et al

\section{Ethical Clearance :}

This study was approved by the Ethical Review Committee of Dhaka Medical College, Dhaka.

\section{Results:}

The mean initial anteroposterior diameter (table-I, Fig-1) of rat's paw of control group (group-1) was $8.03 \pm 0.04 \mathrm{~mm}$. The mean anteroposterior diameter of the rat's paw in control group after 3 hours of Carrageenan injection was $14.22 \pm 0.15 \mathrm{~mm}$. The mean methanolic aqueous initial- anteroposterior diameter of rats paw of group-II methanolic aqueous fraction of Spinacia Oleracea 200mg/ $\mathrm{kg}$ body weight ) was $8.14 \pm 0.18 \mathrm{~mm}$, whereas the mean anteroposterior diameter after 3 hours of Carrageenan injection was $11.45 \pm 0.20 \mathrm{~mm}$ (table-I). The mean initial anteroposterior diameter of rat's paw of group III ( water extract of Spinacia Oleracea $200 \mathrm{mg} / \mathrm{kg}$ boby weigth ) was $8.16 \pm 0.18 \mathrm{~mm}$ and mean anteroposterior diameter after 3 hours of Carrageenan injection was $11.09 \pm 0.28 \mathrm{~mm}$. The mean initial anteroposterior diameter of rat's paw of group IV ( Aspirin $100 \mathrm{mg} / \mathrm{kg}$ body weight ) was 8.17 $\pm 0.17 \mathrm{~mm}$ and mean anteroposterior diameter after 3 hours of Carrageenan injection was $10.10 \pm 0.16 \mathrm{~mm}$. The mean initial anteroposterior diameter of rat's paw of group $\mathrm{V}$ ( Hydrocortisone $2 \mathrm{mg} / \mathrm{kg}$ body weight) was $8.12 \pm 0.19 \mathrm{~mm}$, and mean anteroposterior diameter after 3 hours of Carrageenan injection was $10.83 \pm 0.17 \mathrm{~mm}$. Finally increase in anteroposerior diameter ( Mean \pm SD ) of rat's paw oedema in group-I, II, III, IV and V were $4.94 \pm 0.43,3.30 \pm 0.36,2.93 \pm 0.24,2.93 \pm$ $0.17,2.17 \pm 0.24 \mathrm{~mm}$ respectively. The percentage of inhibition of oedema formation in group II, III, IV and V were 33\%,40\%, 40\% and $47 \%$ respectively (Fig-3).

Table -I

Comparative effect of different groups after 3 hours of Carrageenan injection.

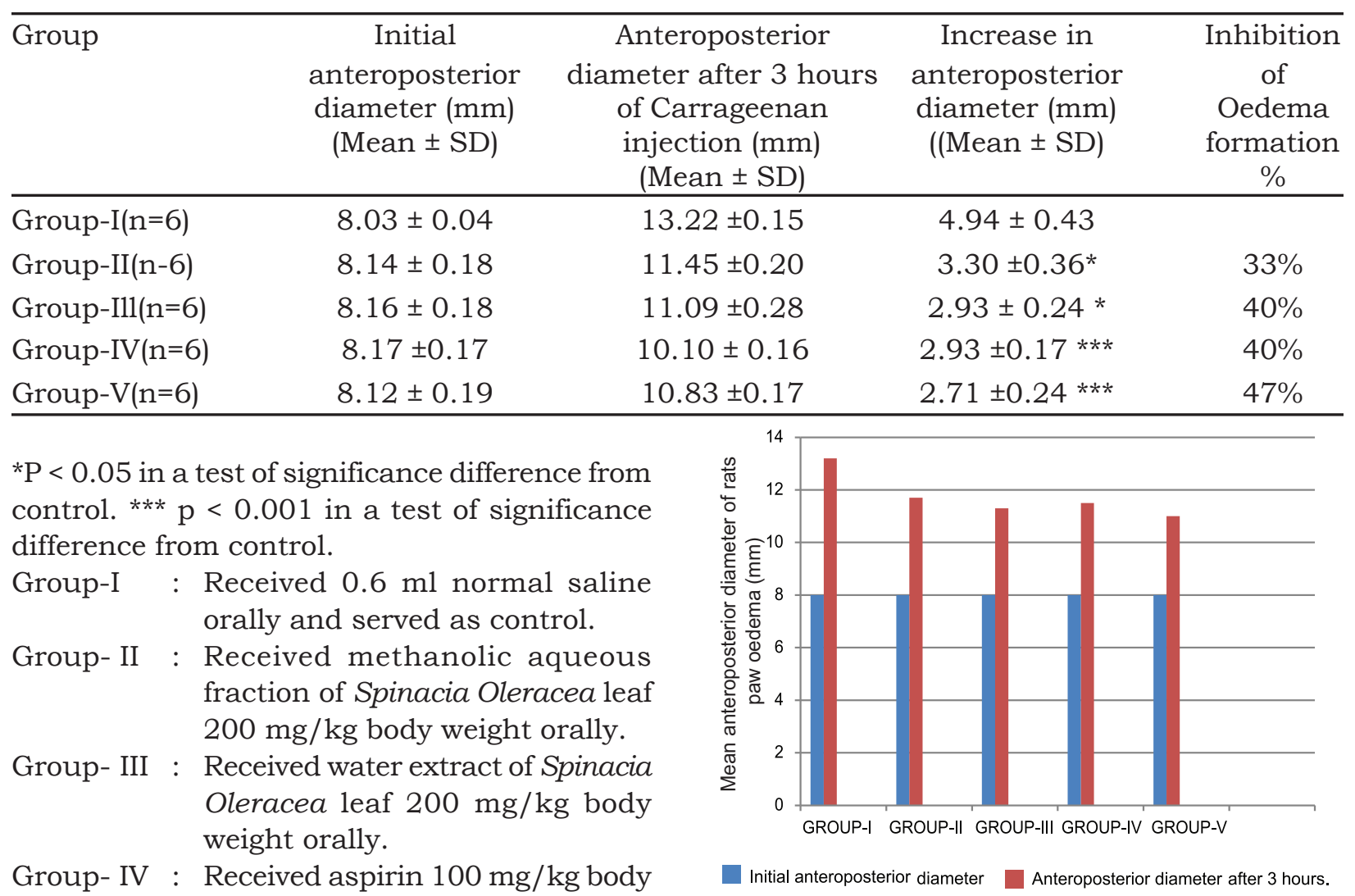

Fig.-2 : Comparative effect of different groups

Group- V : Received hydrocortisone: $2 \mathrm{mg} /$ kg body weight oraly.

after 3 hours of Carrageenan injection. \% of inhibition of inflammation (oedema) 


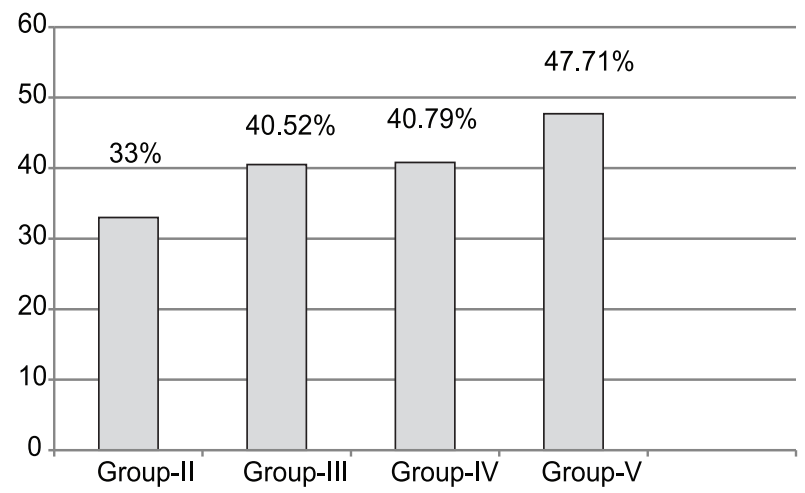

Fig.-3 : Percentage of inhibition of Carrageenan induced in flammation by different does of methanolic aqueous and water extract of Spinacia Oleracea leaf, aspirin and hydrocortisone in comparison to control.

\section{Discussion :}

The present study was carried out to evaluate the anti-inflammatory effect of Spinacia Oleracea leaf. Its anti-inflammatory effects were tested on adult male albino rat. The acute inflammation was induced by injection of $0.1 \mathrm{ml}$ of $1 \%$ Carrageen an suspension in normal saline into the plantar surface of the hind paw of the rats. Administration of methanol acqueous fraction and water extract of Spinacia Oleracea leaf a dose of $200 \mathrm{mg} / \mathrm{kg}$ body weight and $200 \mathrm{mg} / \mathrm{kg}$ body weight orally produced a significant $(\mathrm{P}<0.05)$ anti-inflammatory effect, where the percentage of inhibition of edema formation was $28.75 \%$ and $40.79 \%$ respectively. The effect is dose dependent. Following the administration of aspirin and hydrocortisone the anti-inflammatory effects were highly significant $(\mathrm{P}<0.05)$ and the percentage of inhibition of edema formation were $40.52 \%$ in aspirin and $47.71 \%$ in hydrocortisone. From the result it was observed that the effect of at dose level of $200 \mathrm{mg} / \mathrm{kg}$ body weight a- water extract is better than methanolic aquesous ftaction of Spinacia Oleracea (table-I).

The development of edema in the paw of the rat after the injection of Carrageenan is due to the release of histamine, serotonin and prostaglandins like substances. According to Vinegar et $\mathrm{al}^{5}$, the Carrageen an induced paw edema is a biphasic event. The first phase occur within an hour and is partly due to the trauma of injection and also attributed to the release of histamine, serotonin and kinins. The second phase is sensitive to most clinically effective anti-inflammatory drugs. The second phase of edema is due to the release of prostaglandins play a major role in the development of second phase of reaction, which is measured, after 3 hours of inflammation induction. Treatment with Spinacia Oleracea methanolic aqueres fraction at doses of $200 \mathrm{mg} / \mathrm{kg}$ body weight orally daily produced significant $(\mathrm{P}<0.05)$ antiinflammatory effect and water extract of Spinacia Oleracea leaf at a doses of $200 \mathrm{mg} / \mathrm{kg}$ body weight orally daily produced significant $(\mathrm{P}<0.01)$ anti-inflammatory effect and the percentage of inhibition of granuloma formation were $19.30 \%$ and $27.06 \%$ respectively ${ }^{6}$. Following administration of aspirin and hydrocortisone gave also highly significant $\mathrm{P}<0.001$ anti-inflammatory effects and the percentage of inhibition of granuloma formation were $27.67 \%$ and $38.58 \%$ respectively. The water extract Spinacia Oleracea possesses good anti-inflammatory activity along with wound healing property ${ }^{7}$. Banerjee et al observed similar anti inflammatory effect by Carrageenam induced rat paw oedema test ${ }^{8}$.

The current study was basically pharmacological one and both the modem drugs and herbal products were used to influence the biological system. It was evident that the biological systems have certain limitations, like individual variations, interference in the response with the system, variability in methods and other factors, which might have interfered with primary findings. However, the results obtained in this experiment may not represent the exact effect. Despite all these limitations, interpretation of the results obtained in this study was made carefully and cautiously.

\section{Conclusion :}

This study provide an initial step on demonstrating the anti-inflammatory effect of methanolic aqueous fraction and water extract of Spinacia Oleracea leaf in anti-inflammatory state. The obtained data support the basis for future use of Spinacia Oleracea in traditional system of medicine. Thus, it could be a new agent in reducing morbidity and mortality resulting from inflammatory disease condition. 
The Anti- Inflammatory Effects of Spinacia Oleracea Leaf Extract on Carrageenan Induced Inflammation Yesmin S et al

The findings presented here provide a baseline for future studies designed to quantify the effects of methanolic aquoeous fraction and water extract of Spinacia Oleracea leaf.

\section{References :}

1. Mohan H. Text Book of Pathology, $4^{\text {th }}$ ed. New Delhi, Jaypee Brothers Medical Publisher (Pvt) Ltd; 2000:114-60.

2. Robbins and Cotran. Acute and Chronic inflammation: Pathologic basis of disease. Published by Elsevier, a division of Reed Elsevier India Private Limited; 2007: 31-58.

3. Arora RB, Basu N, Kapoor V and Jain AP. Antiinflammatory Studies on Curcuma Longa (Turmeric). Indian J Med Res. 1971;59:1289-95.

4. Winter CA, Risley EA, Nuss GW. Carrageenam Induced Edema in Hind Paw of the Rat as an Assay for Antiinflammatory Drug. PSEBM. 1962; 111 : 544-47.

5. Vinegar R, Schireiber W, Hugo R. Biphasic Development of Carrageenam Edema in Rats. J Pharmacol Exp. Tehn. 1969; 166: 96-103.

6. Nagar A, Kumar A, Bigoniya P. Anti-inflammatory Potential of Spinacect Oleracea leaf extract. Indian J Phar. 2011; 2(2):80-7.

7. Heo JC, Park CH, Lee HJ, Kim TH. Amelioration of Asthmatic Inflammation by an Aqueous Extract of Spinacea Oleracea. Int J Mol Med. 2010; 25: 409-14.

8. Banerjee S, Sur TK, Mundal S, Das PC, Sikdan S. assessment of the anti-inflammatory effect of Swertia Chitara in Acute and Chronic Experimental Models in Male Albino Rats. Indian Journal of Pharmacol. 2000; 32: 21-4. 